\title{
Responsabilidad social corporativa en empresas audiovisuales españolas. Análisis de RTVE, Atresmedia y Mediaset
}

\author{
Corporate social responsibility in Spanish audiovisual \\ corporations. An analysis of RTVE, Atresmedia and Mediaset
}

Díaz Campo, J. y Berzosa, J.1

Recibido: 9-11-2019 - Aceptado: 8-02-2020

DOI: https://doi.org/10.26441/RC19.1-2020-A6

RESUMEN: Esta investigación analiza las memorias de responsabilidad social corporativa (RSC) de las principales empresas audiovisuales en España (Radio Televisión Española, Atresmedia y Mediaset España). El principal objetivo es determinar en cuál es el nivel de implantación de RSC en cada una de ellas. La metodología utilizada es el análisis de contenido. La ficha empleada está compuesta por 14 indicadores y se basa en el trabajo de Campos (2013). Los resultados muestran una mayor implantación en el medio público que en los privados.

Palabras clave: responsabilidad social corporativa; memorias de responsabilidad social; sostenibilidad; empresas audiovisuales; televisión.

ABSTRACT: This research analyzes the corporate social responsibility (CSR) reports of the main audiovisual companies in Spain (Radio Televisión Española, Atresmedia and Mediaset España). The main objective is to determine the level of implementation of CSR policies in each one of them. We use content analysis methodology. It consists of 14 indicators and it is based of one previous research (Campos, 2013). The results show that there is a greater level of implementation in the public corporation than in the private ones.

Keywords: corporate social responsibility; social responsibility reports; sustainability; audiovisual corporations; television.

\footnotetext{
1 Jesús Díaz Campo es Doctor en Comunicación por la Universidad Complutense de Madrid y Profesor Titular en la Facultad de Empresa y Comunicación de la Universidad Internacional de La Rioja. Sus principales líneas de investigación se centran en Ética de la Comunicación, Responsabilidad Social Corporativa, Comunicación Política y Redes Sociales. jesus.diaz@unir.net, https://orcid.org/0000-0001-5014-8749

Jesús Berzosa es Máster en Comunicación e Identidad Corporativa por la Universidad Internacional de la Rioja e Investigador predoctoral en la Facultad de Empresa y Comunicación de la misma universidad. jberzos@gmail.com, https://orcid.org/0000-0003-2416-4766
} 


\section{Introducción}

La responsabilidad social corporativa (RSC) es un concepto que ha experimentado un claro auge en los últimos años en el mundo empresarial (Carroll, 2015) y el sector de los medios de comunicación no es una excepción. Las compañías se han afanado por implementar mecanismos, herramientas y acciones responsables en un esfuerzo que, a su vez, han tratado y tratan de difundir y hacer llegar a sus distintos grupos de interés, para que estos sean conscientes de las prácticas responsables que llevan a cabo y de los diferentes compromisos que adquieren con y ante la sociedad.

En ese sentido, los stakeholders de cualquier compañía son perfectamente conscientes de que hoy por hoy una empresa socialmente responsable y comprometida con el medio ambiente goza de una mejor reputación. Por tanto, invertir en acciones de responsabilidad social es algo que también puede generar beneficios a medio y largo plazo.

Una de las herramientas fundamentales para realizar este tipo de comunicación entre empresa y grupos de interés es internet y, en general, las nuevas tecnologías, que cada vez están más implantadas en la sociedad y a las que recurren los grupos de interés para consultar y participar. Empleados, clientes, proveedores, administraciones públicas y, en definitiva, todos aquellos que se relacionan directamente o indirectamente con las empresas disponen así de un canal que favorece la interactividad y que les facilita mucho la labor de hacer llegar su feedback a la empresa y mostrarles si están de acuerdo o en desacuerdo con las diferentes acciones.

A su vez, uno de los mecanismos más habituales para que la empresa informe de sus políticas y acciones de RSC son las denominadas memorias de responsabilidad social corporativa, documentos que suelen hacer públicos precisamente a través de sus páginas web corporativas con una periodicidad anual y en las que, a través de distintos apartados, recogen toda esta información.

Pues bien, el objetivo general de esta investigación es analizar las memorias anuales de responsabilidad social corporativa de los tres principales conglomerados audiovisuales en España, para comprobar en qué medida están implantando estándares, medidas y herramientas de responsabilidad social corporativa.

\section{Marco referencial}

Se puede definir la responsabilidad social corporativa (RSC) como:

...el compromiso continuo por parte de las empresas para comportarse de una manera ética y contribuir al desarrollo económico sostenible, al mismo tiempo que se mejora la calidad de vida de los trabajadores y sus familias, así como de la comunidad local y de la sociedad en general (World Business Council For Sustainable Development, 2002: 10).

La RSC constituye uno de los principales activos intangibles de la empresa, y tiene una incidencia significativa en múltiples aspectos, como la evolución de su reputación (Villafañe, 2004a; Truñó Gual \& Rialp Criado, 2008; Vila-López, Küster-Boluda, \& Bigné, 2013; Morales-Blanco-Steger \& Fuente-Cobo, 2018), la imagen corporativa (Orozco Toro, 2017; Costa-Sánchez \& Guarinos-Galán, 2018) o la intención del compra del consumidor, quien añade criterios sociales o medioambientales a los estrictamente económicos a la hora de tomar una decisión (Bigne-Alcañiz \& Currás Pérez, 2008).

Al mismo tiempo, las empresas tratan de hacer llegar estas políticas a sus públicos y encuentran en las páginas web un canal ideal para dar a conocer su información corporativa y sus actuaciones en materia RSC. Así, Tapia \& Caerlos (2014) revelaron que, en España, las empresas que constituyen el Ibex35, es decir, el principal índice de referencia de la bolsa española, considera Internet como 
un canal significativo en su comunicación corporativa y detectó que un $80 \%$ de estas empresas disponían de apartados específicos sobre RSC en sus páginas web.

Las memorias de responsabilidad social corporativa son uno de los instrumentos más importantes para recoger esa información, que incluye actividades e iniciativas, compromisos medioambientales, criterios empresariales y posibles acciones futuras, entre otros aspectos relacionados con el compromiso social de la empresa. Aunque constituye una información no vinculante, supone un gesto de transparencia de la organización que, de hecho, trata de hacerla lo más accesible posible subiéndolo a su página web, de manera que pueda ser consultado libremente por cualquier persona interesada (Moneva, 2005).

Las memorias suelen seguir las pautas marcadas por el Global Reporting Initiative (GRI), una herramienta de verificación mediante cuestionario, que busca promover la transparencia sobre la contribución que las empresas hacen al desarrollo sostenible. Esta recogida de datos se lleva a cabo con la participación de distintos grupos de interés.

\section{RSC y medios de comunicación}

Los medios de comunicación constituyen, por sus propias características, un colectivo de especial interés en el campo de la RSC. En tanto que canal utilizado por las compañías para difundir sus mensajes, se erigen en uno de los principales grupos de interés de cualquier empresa (Berger \& Luckmann, 1984; Villafañe, 2004b; Sweeney \& Coughland, 2008; Cahan, Chen, Chen \& Nguyen, 2015; Fernández Lombao, 2015; Lee \& Riffe, 2017; Tuñez-Lopez \& Costa-Sanchez, 2018) y, por ello, su actuación recibe una atención especial desde el ámbito de la comunicación corporativa en general y la RSC en particular

En ese sentido, son muchas las empresas de comunicación que han desarrollado una cultura de la responsabilidad social corporativa y han implantado políticas de sostenibilidad y RSC en las que, además, han tratado de implicar a sus stakeholders. Así, hay países como Reino Unido o Alemania cuyas radiotelevisiones públicas "asumen la RSC como un modelo de gestión empresarial, tal y como se desprende de las memorias que se publican en sus sitios web" (Fernández \& Campos, 2013:146). No obstante, otros trabajos (por ejemplo, Arévalo Martínez y Cancelo Sanmartín, 2018), alertan sobre una concepción de la responsabilidad social que no incluye aspectos específicos propios de los medios de comunicación, a diferencia de lo que ocurre, según esos mismos análisis, en América Latina.

Además del hecho de que reciban dinero de todos los contribuyentes, algunos autores aluden a asuntos polémicos, como las presuntas escuchas telefónicas ilegales a miembros de la Casa Real, entre otras personas públicas, con la posible implicación de medios de comunicación británicos (Campos, 2013).

En España, las grandes corporaciones llevan ya unos años elaborando memorias de responsabilidad social corporativa. Así, Mediaset lo hace desde 2005, mientras que Atresmedia y RTVE comenzaron a hacerlo en 2007. En esa línea, uno de los hitos que ha marcado un punto de inflexión ha sido la aprobación de la Ley de Economía Sostenible, que establece la obligación para empresas públicas y privadas de presentar anualmente informes de gobierno corporativo y memorias de sostenibilidad y les insta a favorecer la adopción de principios y prácticas de RSC por parte de sus proveedores. Comenzaba así a generalizarse la adopción de planes de RSC, que ya había tenido algún que otro antecedente como el de la EITB del País Vasco (Campos, 2013).

No obstante, López Cepeda \& Manfredi (2013) alertan sobre una carencia a la hora de controlar la aplicación de estas prácticas, la inexistencia en España de un organismo independiente que pueda 
examinar la manera en la que se llevan o no a efecto, si bien reconocen que la publicación de la Ley 7/2010, General de la Comunicación Audiovisual supone una avance en ese sentido, al regular precisamente el derecho a la transparencia que tienen todos los ciudadanos frente a los operadores audiovisuales.

López Cepeda (2011) revela otra carencia relacionada con el perfil de las personas responsables de implementar este tipo de medidas, especialmente en las radiotelevisiones públicas, por cuanto la mayoría de ellas están dirigidas por cargos de naturaleza política y no estrictamente profesional. En la misma línea, Núñez Ladeveze, Irisarri \& Morales (2015) destacan que las televisiones privadas españolas recurren a incluir baremos genéricos en estos documentos informativos para simular un cumplimiento responsable que en realidad se evita cumplir.

Asimismo, diversos análisis de las memorias de RSC de las principales cadenas generalistas de televisión en España, como TVE, Antena 3, Cuatro, Telecinco y La Sexta (Delgado, 2012; Delgado \& Olarte, 2012) coinciden en que el grado de cumplimiento de las políticas y criterios de RSC por parte de estas compañías resulta insuficiente.

No obstante, otros trabajos (Sánchez, 2001; Moreno \& Capriotti, 2006; Medina \& Ojer, 2009; Orozco \& Ferre, 2011; Sierra \& Pascual, 2012; Ferre \& Orozco, 2014; Díaz-Campo, 2014; Aguilar-Conde y Cantalapiedra-Nieto, 2016) indican que el desarrollo de la RSC en el sector de la televisión en España vive una etapa de crecimiento que califican de incipiente, sobre todo si se compara con la situación en otros sectores industriales.

Una situación similar a la registrada en otros países europeos donde las corporaciones de radiotelevisión pública de Alemania, Reino Unido o Irlanda, entre otros, han comenzado a comunicar sus acciones de RSC en los últimos años, si bien aparecen carencias en su aplicación y no hay un seguimiento exhaustivo de la normativa internacional. (Gulyás, 2009; Fernández \& Campos, 2013; Sandoval, 2013). Ingenhoff \& Koelling (2012) apuntan a una mayor presencia de herramientas y actividades de RSC en las corporaciones públicas que en los medios privados en Europa, mientras que Bonsón \& Escobar (2006) ponen el acento en el incremento de la transparencia de las compañías promovido desde la propia Unión Europea.

\section{Metodología}

La primera fase de la investigación consistió en el diseño de una ficha metodológica, que se basó en la utilizada por Campos (2013) a partir de los estándares del Global Reporting Initiative, con algunos cambios para adaptarse a las características concretas de las memorias estudiadas. Así se conformó una ficha compuesta por 14 indicadores:

1. Transparencia de datos sobre la propiedad

2. Principios editoriales

3. Número y cuantía de las ayudas públicas recibidas

4. Ingresos de publicidad

5. Otros sistemas de financiación

6. Cuestiones relacionadas con la libertad de expresión

7. Cuestiones medioambientales (consumo de papel, tinta, etc.)

8. Promoción de la creatividad y el talento local 


\section{Actuaciones de $\mathrm{I}+\mathrm{d}+\mathrm{i}$}

10. Verificación de las cifras de circulación y audiencia

11. Accesibilidad. Este indicador hace referencia la existencia o no de acciones referidas a personas con discapacidad auditiva (subtítulos, etc.)

12. Misión, visión, valores

13. Campañas de responsabilidad social corporativa

14. Campañas de voluntariado

El siguiente paso fue seleccionar las empresas que serían analizadas. A partir de los estudios de referencia en España en cuanto a datos de audiencia (Kantar Media y Estudio General de Medios) y volumen de negocio (diario digital económico eleconomista.es) se creó un universo compuesto por las tres principales empresas de comunicación, que agrupan a las cinco principales cadenas de televisión generalistas:

- Radio Televisión Española (RTVE). Grupo empresarial de titularidad pública que comprende dos canales generalistas como La 1 y La 2, canales temáticos informativos, deportivos e infantiles, y Radio Nacional de España, que agrupa a distintas cadenas de radio.

- Atresmedia. Grupo privado que engloba dos canales generalistas de televisión como Antena 3 y La Sexta, diversos canales temáticos que emiten a través del sistema de Televisión Digital Terrestre (TDT) y la cadena radiofónica Onda cero.

- Mediaset España. Comprende dos canales generalistas de televisión (Telecinco y Cuatro) y varios canales temáticos más.

A partir de ahí, la metodología empleada fue el análisis de contenido de las memorias de 2015, puesto que, en el momento de realizar la investigación, Mediaset España y Atresmedia habían publicado la de 2016, pero no así RTVE. Previamente, a modo de pretest se analizó una de las memorias elegida al azar, para comprobar que la ficha metodológica se adecuaba a las características de la investigación.

Se examinaron las tres memorias buscando la presencia de información relativa a los 14 indicadores mencionados, a través de campos semánticos cercanos a la naturaleza dichos indicadores.

Como limitación del estudio, cabe señalar que la investigación se ha limitado al análisis de las memorias de las tres corporaciones, pero que los autores no conocen en detalle el desarrollo de las actividades mencionadas en ellas y, por tanto, no pueden corroborar en qué medida esas memorias constituyen un reflejo más o menos fiel de la realidad.

\section{Análisis de resultados}

A continuación, se presentan los resultados del análisis de contenido de las tres memorias estructurado de acuerdo a los 14 indicadores mencionados. Se ha recogido en cada caso lo que se ha considerado más relevante. No obstante, en las referencias bibliográficas se incluyen los enlaces al texto íntegro de las tres memorias, de forma que el lector que desee profundizar en alguno de esos aspectos pueda hacerlo.

\section{Transparencia de datos sobre la propiedad}

RTVE dispone del departamento "Dirección de Patrimonio", que gestiona sus inmuebles. Entre sus 
principales actuaciones en 2015 destacan las relativas al proceso de modernización del equipamiento; integración de los sistemas de gestión eléctrica y PCI de Torrespaña y Prado del Rey; inicio de las obras de medidas correctoras en Torrespaña; y aprobación y obtención de obras y licitación para la construcción de nuevas edificaciones en Prado del Rey.

Además, realizó actuaciones constructivas dirigidas a corregir la dispersión territorial existente. La memoria señala que esta actuación permitió la optimización de energética mediante la reducción de costes económicos y reduciendo el impacto medioambiental. La memoria recoge también actuaciones en varios centros territoriales. Un dato importante es el compromiso inmobiliario con los trabajadores de la compañía. RTVE se comprometió a 'desamiantar' varias oficinas.

La memoria de Atresmedia no ofrece datos concretos sobre sus propiedades. Este grupo dispone de varios edificios centrales en San Sebastián de los Reyes (Madrid). La única información de este tipo que se puede encontrar en la memoria hace referencia a su eficiencia energética y el cambio de luminaria de fluorescencia por tecnología LED para poder ahorrar tanto en la factura de la luz como en emisiones medioambientales.

Por su parte, Mediaset España tampoco aporta información sobre sus propiedades. Únicamente hay referencias a este apartado dentro del cuestionario GRI G4, en el que indica que: "las instalaciones gestionadas directamente por el Grupo se encuentran en polígonos industriales o zonas urbanas alejadas de espacios naturales protegidos" (Mediaset España, 2016:190).

Los datos aluden directamente a la gestión energética de los bienes inmuebles con políticas de ahorro energético y costes para la empresa con medidas de renovación del alumbrado de las instalaciones, sustituyendo los aparatos de iluminación tradicional por LED, y generando una reducción en el consumo de alrededor de $50.000 \mathrm{KWh}$ anuales.

\section{Principios editoriales}

RTVE dispone de un régimen jurídico que garantiza su independencia, neutralidad y objetividad. Los trabajadores de RTVE y sus principios editoriales defienden la libertad de expresión, los valores constitucionales que garantizan la libertad, igualdad y pluralidad e independencia del poder ejecutivo. Sus principios básicos de la programación son públicos, cualquier ciudadano puede acceder a ellos. RTVE dispone de la figura de la Oficina del Defensor del Espectador, cuyo objetivo es ser el enlace entre los ciudadanos y los responsables de la corporación.

Atresmedia destaca que ofrece contenidos de calidad, accesibles, que reflejan las diferentes realidades, entornos y voces que componen la sociedad y que respondan a las necesidades y expectativas de la audiencia. Por ello, apuestan por un modelo basado en la calidad, la diversidad de géneros, la pluralidad, la innovación y el compromiso. Por otro lado, dispone de un mecanismo de control de contenidos para garantizar que las emisiones se ajustan a los valores, códigos éticos y políticas del grupo.

Mediaset España incorpora el epígrafe "Gestión de los contenidos", que hace referencia a la pluralidad y la diversidad de las fuentes de información, la libertad de expresión de independencia periodística, la imparcialidad informativa, la aplicación de estándares éticos que regulan la actividad, junto con la participación ciudadana y la accesibilidad a los contenidos. Además indican que en su grupo prevalece la función del entretenimiento.

También mencionan los principios de la actividad periodística. Disponen de un Código Ético para que los periodistas cumplan estándares como respetar la veracidad, objetividad e independencia en la información que transmiten. Por otra parte, se compromete a rectificar la información que no sea veraz y a integrar todas las sensibilidades e ideas políticas en sus informativos y distintas tertulias que se realizan en sus programas. 


\section{Número y cuantía de las ayudas públicas recibidas}

La corporación RTVE es totalmente pública y recibe la mayoría de sus ingresos de los Presupuestos Generales del Estado del Gobierno de España. En 2015 aportó 281,4 millones de euros por esta vía. Las cuentas arrojaron unas pérdidas que ascendieron a 37,8 millones de euros.

Por su parte, la memoria de Atresmedia hace una mínima referencia en el cuadro económico. Recibió de la Administración Pública 6,4 millones de euros. Igualmente, se menciona la existencia de reuniones con la Administración y con los organismos reguladores, y su colaboración en cuestiones relacionadas con hábitos saludables.

Mientras, Mediaset España ofrece datos detallados sobre las subvenciones recibidas. Dispone de una sección denominada "Fiscalidad" donde desgranan los conceptos correspondientes. Según su memoria, recibe el 100\% de ayudas por parte del Instituto de la Cinematografía y de las Artes Audiovisuales para la actividad de coproducción cinematográfica, que se destina a su filial, Mediaset Cinema. En 2015, estas ayudas ascendieron a 706.820 euros. Sin embargo, en el apartado "otras ayudas recibidas de organismos públicos", se indica que no se recibió ninguna cantidad.

\section{Ingresos de publicidad}

RTVE no dispone de ingresos por publicidad desde 2009. Desde entonces, por asignación gubernamental, recibe ingresos de los operadores de telecomunicaciones y de televisión, entre ellos Atresmedia y Mediaset. RTVE renunció a la publicidad comercial en sus cadenas a cambio de una financiación mediante tasas, a los canales privados en abierto con el 3\% de sus ingresos brutos. Los canales de pago abonan el 1,5\% y las empresas de telecomunicación el 0,9\%. En 2015, estos ingresos ascendieron 183,8 millones de euros.

Atresmedia no ofrece datos exactos, aunque refleja que su comercializadora ha obtenido una cuota del $19,3 \%$ del total de la inversión en medios convencionales y existe otra referencia dentro de los "Resultados financieros", en los cuales indican que, a nivel global, el mercado de la publicidad en medios convencionales alcanzó los 5.016,7 millones de euros en 2015, un 7,5\% más que el año anterior. Mientras, en el índice de contenido GRI G4 indican que el 93\% de sus ingresos provienen de publicidad.

Mediaset España ofrece detalles en su apartado de "Modelo de negocio" y "Principales Indicadores de Desempeño (KPIs)", en la que indican que los ingresos publicitarios netos que recibieron fueron 902 millones de euros.

\section{Otros sistemas de financiación}

Las memorias no recogen mucha información relativa a este apartado. RTVE indica que se contabilizaron ingresos procedentes de liquidaciones complementarias de operadores emitidas por la Comisión Nacional del Mercado de Competencia (CNMC) por un importe total de 12,7 millones de euros.

Por su parte, Atresmedia cifra sus ingresos netos en 970,2 millones de euros, pero no se indica explícitamente, sino que hacen balance de los ingresos producidos por sus divisiones de televisión, radio, publicidad, cine, música, etc. En este sentido, en el cuestionario GRI G4, aluden a que un $7 \%$ de los ingresos provienen de actividades minoritarias como derechos de autor, producciones cinematográficas o acciones especiales.

Mediaset España, desgrana algo más este indicador, además de hacer una comparativa con años anteriores (ver Tabla 1). 
Tabla 1. Ingresos Mediaset España

\begin{tabular}{|l|r|r|r|}
\hline Ingresos (miles de €) & \multicolumn{1}{|c|}{2013} & \multicolumn{1}{|c|}{2014} & \multicolumn{1}{c|}{2015} \\
\hline Publicidad & 766.560 & 855.682 & 897.973 \\
\hline Otros publicidad & 1.737 & 2.424 & 3.815 \\
\hline Prestación servicios & 40.715 & 53.011 & 48.824 \\
\hline Otros & 9.813 & 8.283 & 7.279 \\
\hline Otros ingresos explotación & 7.995 & 12.687 & 14.040 \\
\hline
\end{tabular}

Fuente: Mediaset (2016)

\section{Cuestiones relacionadas con la libertad de expresión}

La Corporación RTVE dispone de la sección "Llegamos a todos con Televisión Española", que incluye el epígrafe "Pluralidad de voces". Alude a los servicios informativos, que dan voz a los representantes de todos los partidos políticos siguiendo la proporcionalidad parlamentaria. Además, se sigue el artículo 9 de la Ley 8/2009, en el que se indica que se debe dedicar al menos 12 horas semanales en horario no residual entre sus distintos canales a emitir programas que den acceso a los distintos colectivos.

Mientras, Atresmedia hace hincapié en que aplica las recomendaciones de responsabilidad corporativa del Código de Buen Gobierno de la Comisión Nacional del Mercado de Valores (CNMV). En referencia a la libertad de expresión, lo destaca como aspecto relevante para los grupos de interés.

Por último, Mediaset España dispone de código ético de obligado cumplimiento por todos los grupos implicados En el mismo, entre otras cuestiones, hacen referencia a la libertad de expresión e independencia periodística. Además, en su modelo de gestión de contenidos, destacan que se debe aplicar la pluralidad, la diversidad de fuentes, la libertad de expresión y la imparcialidad informativa junto a la participación ciudadana y la accesibilidad a los contenidos.

\section{Cuestiones medioambientales}

RTVE alude que su programación incluye espacios como "Aquí en la Tierra", que promueve valores de protección del medio ambiente, la defensa de los animales y la utilización de recursos para promover el reciclaje, así como concienciar a la ciudadanía sobre los desastres naturales, las consecuencias de las catástrofes medioambientales y la propagación de noticias sobre el cambio climático. También se menciona que RTVE emitió programas sobre clima, flora y fauna autóctona, pero no ofrece datos de audiencia.

Por su parte, Atresmedia dedica un apartado a la "Dimensión ambiental", donde detalla el consumo de papel, tóner y cartuchos de impresión, luces fluorescentes, residuos sólidos urbanos, consumo de gas, gasóleo para calefacción, electricidad, agua, emisiones directas de CO2. Asimismo, indican que cumplieron los compromisos de mejora ambiental.

Mediaset España también dedica un apartado a este indicador. Indica que su actividad no genera impactos ambientales directos significativos, si bien por el hecho de desarrollar una actividad económica y dada la magnitud de la compañía, se produce un cierto impacto ambiental de forma colateral. (Mediaset España, 2015:162). Además, ofrece datos concretos y detallados sobre su consumo en agua, energía y materiales.

Un dato muy interesante es que la compañía hace pública la información para demostrar que está concienciada con esta problemática. Para ello informa sobre los datos de emisión sobre vertidos, residuos en base al GreenHouse Gas Protocol Corporate Standard, emitido por el World Business Council for Sustainable Development (WBCSD) y el World Resources Institute(WRI). 


\section{Promoción de la creatividad y del talento local}

RTVE dispone del Instituto RTVE para este fin. Tiene 40 años de bagaje y en el mismo tiene alumnos que forma para que puedan crear contenidos, guiones televisivos, radiofónicos y ser futuros profesionales de RTVE o de otra empresa audiovisual. En la memoria hacen referencia a que, además de formación, prestan servicios de carácter cultural y social.

Además, RTVE dispone de Orquesta Sinfónica. Se trata de una orquesta de gran prestigio y que en 2015 cumplió 50 años de existencia. En sus actuaciones tiene una gran acogida de público y de audiencia en televisión y radio.

Por su parte, Atresmedia apuesta por la creatividad y dispone de la sección "Crea Cultura", donde su reto es el talento artístico y promueve el respeto de la propiedad intelectual. También, en noviembre de 2005, lanzó Flooxer, una plataforma para promover el talento digital para las marcas y sus creadores atrayendo, según la entidad, 770.000 usuarios únicos. Por otro lado, dispone de Atresmedia Cine, donde se da cabida a los realizadores noveles y nuevos directores que buscan una oportunidad dentro del séptimo arte. Atresmedia dispone, al igual que RTVE de formación de universitarios con post-grados, así como programas de talento y formación interna para sus propios empleados.

En este sentido, Mediaset España impulsa el desarrollo sostenible de su proyecto empresarial y pretende contar con personas que tengan talento y sean capaces de desarrollar contenidos que atraigan a la audiencia. Dispone de una política de formación para sus trabajadores en aras de que manejen las nuevas tecnologías audiovisuales y perfeccionen sus aptitudes. Mediaset España no cuenta con formación propia oficial como sí tiene RTVE, sin embargo, sí tiene acuerdos con centros universitarios. Destaca la selección de los mejores estudiantes del Máster Universitario de Creación y Gestión de Contenidos Audiovisuales que imparte la Universidad Europea de Madrid.

\section{Actuaciones de $\mathrm{I}+\mathrm{d}+\mathrm{i}$}

RTVE vincula estas cuestiones con su rediseño en internet. Así por ejemplo, impulsa en España el estándar HbbTV para televisores inteligente que pueden ver contenidos bajo demanda. Asimismo, se apoya en la Ultra Alta Definición o UHD 4k. También se afanan por llevar la ficción, no solo a la pantalla, sino a las redes sociales, con un exponente muy claro en la serie "El Ministerio del Tiempo". Destaca que ofreció la posibilidad de utilizar la multiseñal con motivo de los "Premios Goya". El usuario de la web pudo ejercer de realizador y elegir la cámara que quiso ver. En la web dispuso de contenidos denominados " 360 "”.

En Atresmedia destaca el "Compromiso Atresmedia" que promueve, entre otras cuestiones, la investigación científica en España. Dentro de este campo y junto a la Fundación AXA, logró que pusiera en marcha la Agencia Estatal de Investigación a través de una recogida de firmas que difundió a través de sus medios de comunicación.

En el caso de Mediaset España, indica que apoyan la innovación para crear sus contenidos audiovisuales. Para ello dispone de áreas de contacto con las productoras que le nutren de contenidos y distribuidoras en aras de llevar a la cadena de Fuencarral, las novedades tecnológicas y las distintas tendencias en el mundo de la televisión. De hecho, disponen de un departamento que verifica y aprueba estas mejoras.

\section{Verificación de las cifras de circulación y audiencia}

Las tres memorias hacen referencia a esta cuestión. En el caso de RTVE, aluden a que los "Telediarios" llegaron a estar en segunda posición en 2015 con una cuota de pantalla global del 13,5\%. También destacan debates electorales, ficción, entretenimiento y magazines y retransmisiones es- 
peciales, como la habitual "Lotería de Navidad" o el "Festival de Eurovisión". Además, recoge los datos de audiencia de Radio Nacional de España.

Por su parte, Atresmedia indica que en 2015 obtuvieron un 26,8\% de media anual de cuota de pantalla. Destacan sus programas informativos, como debates electorales, los programas de entretenimiento y hacen referencia a que la Champions League de fútbol llegó a sus pantallas en septiembre de 2015. También recogen los datos de sus diferentes cadenas de radio, que sumaron casi cinco millones de seguidores.

Mediaset destaca que en 2015 consiguió un 31\% de cuota de audiencia. Menciona que supera en 4.2 puntos a su inmediato competidor, Atresmedia. Su canal que más audiencia individual alcanza es Telecinco con un promedio del $14.8 \%$. Hace referencia también a otros canales y remarcan que los temáticos son los más vistos, si bien no ofrecen datos de verificación externa en la memoria.

\section{Accesibilidad}

RTVE detalla que promovió en su programación espacios por la integración en programas como "Para todos La 2", "Con mis ojos", dedicado a los invidentes y con la colaboración de la empresa ONCE. Asimismo, la memoria recoge que se esfuerza por atender a las personas afectadas por algún tipo de discapacidad, dedicando multitud de horas de programación a que personas con problemas de audición o visión pudieran, mediante subtítulos, audio-descripción y lenguaje de símbolos, poder seguir la programación de la televisión pública. Indica que es un compromiso ineludible. Todas estas iniciativas llegaron también a la web de RTVE donde se subtituló el 80\% de la programación.

Atresmedia, por su parte, invirtió en accesibilidad en todas las plataformas, especialmente en su aplicación Atresplayer. Así lo refleja en su "visión". En la misma indica que una de sus premisas es impulsar la accesibilidad a los contenidos audiovisuales.

Por último, desde Mediaset España, refleja en su memoria y dentro de la gestión de contenidos, información sobre la accesibilidad. Para esta empresa la accesibilidad de los contenidos constituye un aspecto fundamental de la responsabilidad de los medios de comunicación.

\section{Misión, visión, valores}

RTVE dedica un apartado especial que denomina “Así somos". En referencia a la misión, indican que RTVE responde al carácter de empresa de servicio público, por lo que debe ofrecer una información rigurosa, independiente y plural, y un entretenimiento de calidad; así como fomentar el debate, la innovación y la creación. Asimismo, apoyan la difusión de las artes, la ciencia y la cultura. También apelan a dar cauce a la participación.

En cuanto a la visión aluden de nuevo a su función como servicio público. En multitud de ocasiones hacen referencia a este término, pero también quieren ser el medio de comunicación de referencia en nuestro país. Se comprometen, además de con el sentimiento nacional, con las autonomías. Además, aluden a una visión global para difundir, a través de su medio, los valores constitucionales. Por último, los valores de RTVE hacen referencia a los de la libertad, igualdad, pluralismo y tolerancia, sobre los que se asienta la convivencia democrática.

Por lo que respecta a Atresmedia, su misión consiste en difundir una oferta informativa y de entretenimiento diversa, innovadora y de calidad, para responder a sus grupos de interés y lograr la confianza, combinando el liderazgo y la sostenibilidad. En cuanto a la visión, su objetivo es ser un grupo de referencia y contribuir de forma positiva y valiosa en la sociedad en la que operan. Mientras los valores hacen referencia al compromiso, la calidad, la creatividad, la visión a largo plazo, la innovación, el liderazgo y el trabajo en equipo. 
Por su parte, Mediaset España no menciona su visión, misión y valores como tales en ninguno de sus apartados de su memoria de RSC. Hace alusiones puntuales dentro de su apartado "Modelo de gestión de los contenidos", donde destacan que la pluralidad y la diversidad de fuentes de información, así como la participación activa de diversos sectores de la sociedad, son valores a los que presta gran atención. También en su apartado: "Modelo de gobierno", indican que la libertad de expresión, el pluralismo y la diversidad, son los valores corporativos.

\section{Campañas de RSC}

RTVE recoge todas las acciones llevadas a cabo. Una vez más hace alusión a su carácter de servicio público y a las actuaciones en materia social, medioambiental y laboral. Por otro lado, se hacen eco de los más de 700 espacios vinculados a los ocho Objetivos de Desarrollo del Milenio de Naciones Unidas, cuya adhesión se hizo en el año 2000.

Como ejemplos de campañas, se realizaron donación de órganos con algunos de los rostros más conocidos de la corporación, recogida de fondos para la investigación del cáncer, homenaje al Maestro para sensibilizar a la población sobre este colectivo, promoción de la lectura, potenciación de la Lengua Española, intercambio de libros de textos a favor de la cultura y la sostenibilidad, apoyo a las personas mayores a través de los programas con consejos preventivos y de seguridad, compromiso con la discapacidad, programas de concienciación de conciliación familiar y laboral evitando que los programas terminarán más allá de las doce de la noche, entre otras.

Por su parte, Atresmedia destaca que los espectadores valoran sus acciones de RSC (Atresmedia, 2016: 86). Destacan "Ponle Freno", dedicada a reducir la mortalidad en las carreteras a través de consejos para el conductor; "Hazte Eco", para ayudar a sensibilizar al público más joven con campañas de reciclaje y respeto con el medio ambiente; "Objetivo Bienestar", dedicadas a promover la alimentación sana y equilibrada para los más pequeños en los colegios y reducir la tasa de sobrepeso que atesora nuestro país, o "Constantes y Vitales", que persigue mejorar la salud de los ciudadanos y promover campañas de donación de órganos. Todas estas campañas se realizan de forma solitaria o con colaboraciones con empresas colaboradoras, como la Fundación Mutua Madrileña.

Por su parte, Mediaset España, tiene en su campaña "12 meses, 12 causas", su mayor estandarte en campañas de RSC. Esta campaña, que engloba todas las acciones de RSC ha recibido el "Premio Chicote" y, dentro de las acciones, destaca "Doy la cara", contra la violencia de género, que recrea casos reales de mujeres y sus familiares víctimas de ataques psíquicos o físicos que consiguieron salir de esta terrible situación.

\section{Campañas de voluntariado}

RTVE dispone su estructura en diversas áreas de actuación. Permite a antiguos empleados jubilados que participen en las campañas. Entre los objetivos destacan acciones en defensa de los derechos humanos, la colaboración con grupos desfavorecidos, apoyo al medio ambiente, lucha contra enfermedades o la desigualdad. Cabe destacar los tres voluntarios de RTVE que estuvieron en Indonesia cuando se cumplieron 10 años del devastador tsunami que asoló al país. Estos trabajadores realizaron un documental titulado "La ola negra".

Atresmedia resalta como principal objetivo contribuir al desarrollo de la sociedad poniendo a disposición de las campañas que lo requieran a empleados de la compañía. Se hacen eco de la campaña "Activa tu Voluntad" en colaboración con diversas entidades dedicadas al voluntariado. Además, señalan que tomaron parte en 16 actividades de voluntariado, colaboraron con 13 entidades sociales diferentes y participaron 99 voluntarios, con un empleo de 2.102 horas de forma altruista. 
Por su parte, Mediaset España, no especifica en su memoria planes o acciones de voluntariado para sus trabajadores, pero alude a que disponen de beneficios sociales recogidos en su convenio colectivo.

\section{Resumen final}

En la Tabla 2 se ha resumido de manera sintética el cumplimiento de los indicadores estudiados por parte de las tres corporaciones. Como puede verse, RTVE registra un cumplimiento del 100\%, mientras que Atresmedia y Mediaset cumplen 11 de los 14 indicadores $(78,6 \%)$ cada una, si bien no son exactamente los mismos los que recogen en cada una de las memorias.

Tabla 2: Cumplimiento de los indicadores por parte de las corporaciones analizadas

\begin{tabular}{|c|c|c|c|}
\hline Indicador & Atresmedia & Mediaset & RTVE \\
\hline Transparencia datos propiedad & & & $x$ \\
\hline Principios editoriales & $x$ & $x$ & $x$ \\
\hline Ayudas públicas recibidas & $\mathrm{x}$ & $\mathrm{x}$ & $\mathrm{x}$ \\
\hline Ingresos publicidad & & $x$ & $\mathrm{x}$ \\
\hline Otros sistemas financiación & & $x$ & $\mathrm{x}$ \\
\hline Cuestiones relacionadas libertad expresión & $x$ & $x$ & $\mathrm{x}$ \\
\hline Cuestiones medioambientales & $\mathrm{x}$ & $\mathrm{x}$ & $\mathrm{x}$ \\
\hline Promoción creatividad y talento local & $\mathrm{x}$ & $\mathrm{x}$ & $x$ \\
\hline Actuaciones de $\mathrm{I}+\mathrm{d}+\mathrm{i}$ & $x$ & $x$ & $\mathrm{x}$ \\
\hline Verificación cifras circulación y audiencia & $x$ & $x$ & $\mathrm{x}$ \\
\hline Accesibilidad & $x$ & $x$ & $\mathrm{x}$ \\
\hline Misión, visión, valores & $x$ & & $x$ \\
\hline Campañas de RSC & $x$ & $x$ & $\mathrm{x}$ \\
\hline Campañas de voluntariado & $x$ & & $x$ \\
\hline Total & 11 & 11 & 14 \\
\hline
\end{tabular}

Fuente: Elaboración propia

\section{Discusión y conclusiones}

Los resultados obtenidos muestran que las principales corporaciones audiovisuales españolas están implantando de manera creciente estándares, medidas y herramientas de RSC. Asimismo, se observa una diferencia importante en el grado de implantación en función del carácter de la empresa: la corporación pública RTVE es la única de las tres analizadas que cumple con todos los indicadores estudiados, algo que no sucede con Atresmedia y Mediaset España. Las cuestiones económicas (financiación, propiedades, etc.) son las que registran una diferencia más significativa.

Por el contrario, parámetros como la libertad de expresión, asuntos relacionados con el medioambiente, innovación o datos de audiencia muestran unos grados de cumplimiento similares, con independencia de que la compañía sea pública o privada.

Por tanto, estos resultados ratifican los obtenidos en otros trabajos previos tanto en España (López-Cepeda \& Manfredi, 2013) como en Europa (Ingenhoff \& Koelling, 2012) que ya concluían la existencia de diferencias significativas en el grado de cumplimiento entre las empresas públicas y las privadas.

Asimismo, en comparación con otros trabajos además de las dos citados (por ejemplo, Delgado Sierra, 2012; Delgado \& Olarte, 2012; Ferré \& Orozco, 2014); se demuestra que las empresas 
audiovisuales, con independencia de su naturaleza pública o privada, siguen apostando de manera creciente con la RSC y aumentan poco a poco el grado de cumplimiento de los diversos parámetros e indicadores, si bien todavía queda un amplio camino por recorrer, especialmente en todo lo relativo a las cuestiones de tipo económico

Del mismo modo, los datos arrojan un panorama más positivo que el descrito por Arévalo Martínez y Cancelo Sanmartín (2018) en cuanto a la concepción de la responsabilidad social corporativa en los medios de comunicación europeos, con una mayor presencia de aspectos directamente relacionados con la naturaleza de estas empresas.

Tratando de desgranar estos aspectos por corporaciones se observan, por un lado, aspectos coincidentes y, por otro, elementos diferenciadores. En el primer caso, las tres corporaciones profundizan en las cuestiones medioambientales o la accesibilidad, dos aspectos en los que las tres alcanzan lo que se puede considerar un nivel de cumplimiento óptimo.

Pero, al mismo tiempo, se detectan diferencias que se traducen, por ejemplo, en una apuesta clara por la transparencia en el caso de RTVE, que se materializa en la información detallada en cuestiones como la misión, visión o valores, los principios editoriales o los datos sobre la propiedad, entre otros. Por su parte, Atresmedia parece poner el acento en lo relativo a las campañas de RSC o de voluntariado, con hitos destacables como la iniciativa "Ponle freno". Mientras, en lo que respecta a Mediaset, destaca especialmente la información referente a cuestiones económicas, como las ayudas recibidas o la publicación de otros sistemas de financiación.

En este sentido, las tres corporaciones combinan un enfoque de gestión, aunque orientado a diferentes aspectos de la RSC, con la puesta en marcha de acciones más puntuales o campañas concretas.

Por último, este trabajo abre la posibilidad de futuras investigaciones que sirvan para comparar los resultados obtenidos con otras corporaciones audiovisuales como puedan ser las radiotelevisiones autonómicas o en otros sectores igualmente relacionados con el mundo de lo audiovisual como, por ejemplo, productoras.

\section{Bibliografía}

Aguilar Conde, P. \& Cantalapiedra Nieto, B. (2016). Un enfoque de la RSC en la televisión en España. Visión comparada a través del análisis de Atresmedia y Mediaset. Gizarte Ekonomiaren Euskal Aldizkaria -Revista Vasca de Economía Social, 13, 97-124, https://doi.org/10.1387/reves.17537

Arévalo Martínez, R. I. y Cancelo Sanmartín, M. (2018). Los medios de comunicación y su responsabilidad social organizacional en Iberoamérica". Estudios sobre el Mensaje Periodístico, 24 (2), 1043-1055. http://dx.doi.org/10.5209/ESMP.62201

Atresmedia (2016). Informe anual y de responsabilidad corporativa 2015. www. atresmediacorporacion.com/documents/2016/04

Berger, P. \& Luckmann, T. (1984). The social construction of reality: A treatise on the sociology of knowledge. London: Penguin Books. ISBN: 9780385058988

Bigne-Alcañiz, E. \& Currás-Pérez, R. (2008). ¿Influye la imagen de responsabilidad social en la intención de compra? El papel de la identificación del consumidor con la empresa. Universia Business Review, 19: 10-23. https://dialnet.unirioja.es/descarga/articulo/2703153.pdf

Bonson, E. \& Escobar, T. (2006). Digital reporting in Eastern Europe: An empiricalstudy. International Journal of accounting informations systems, 7(4), 299-318. https://doi.org/10.1016/j. accinf.2006.09.001 
Cahan, S. F.; Chen, C.; Chen, L. \& Nguyen, N. H. (2015). Corporate social responsibility and media coverage. Journal of Banking \& Finance, 59, 409-422, https://doi.org/10.1016/j. jbankfin.2015.07.004

Campos, F. (2013). Las empresas de comunicación adaptan los estándares de gestión de la Responsabilidad Social Corporativa. Revista de Comunicación, 12, 32-58.

Carroll, A. B. (2015). Corporate social responsibility: The centerpiece of competing and complementary frameworks. Organizational Dynamics, 44(2), 87-96. http://dx.doi.org/10.1016/j. orgdyn.2015.02.002

Costa-Sánchez, C. \& Guarinos Galán, V. (2018). Gestión de marca corporativa online de los canales públicos de televisión en Europa. Propuesta de indicadores para su medición. Revista Latina de Comunicación Social, 73, 895-910. https://doi.org/10.4185/RLCS-2018-1287

Delgado, V. \& Olarte, M. (2012). Responsabilidad social corporativa en el sector de la televisión. Un estudio longitudinal de las memorias de sostenibilidad. Revista Internacional de Investigación en Comunicación Ad Research ESIC, 6(6), 112-129.

Delgado Sierra, V. (2012). Estudio del grado de desarrollo de la responsabilidad social corporativa a través de las memorias de sostenibilidad y de las audiencias en televisión. Tesis Doctoral. Universidad de La Rioja.

Díaz-Campo, J. (2014). Las cadenas de televisión españolas en Internet: un estudio sobre la calidad de sus sitios web. Estudios sobre el Mensaje Periodístico, 20 (1), 67-84. http://dx.doi. org/10.5209/rev_ESMP.2014.v20.n1.45219

Fernández, T. \& Campos, F. (2013). La Responsabilidad Social Corporativa en las radiotelevisiones públicas de Europa. Cuadernos. info, (33), 145-157. http://dx.doi.org/10.7764/ cdi. 33.530

Fernández Lombao, T. (2015). La RSC como garante de la accesibilidad de colectivos vulnerables en las radiotelevisiones públicas europeas. Guadarrama Rico, L. A., Suárez Villegas, J. C. y González Jimenez, M. M. (eds.): Desafios éticos de la comunicación en la era digital: III Congreso Internacional de Ética de la Comunicación, libro de actas. (pp. 352-360). Sevilla: Universidad de Sevilla.

Ferré, C. \& Orozco, A. (2014). La RSC desde la perspectiva de las empresas de comunicación. Recuperado de: $\mathrm{http}: / / \mathrm{www}$. portalcomunicacion.com/lecciones_exp.asp?id=89

Gulyás, A. (2009). Corporate social responsibility in the British media industries preliminary findings. Media, Culture \& Society, 31(4), 657-668. https://doi.org/10.1177/0163443709335281

Ingenhoff, D. \& Koelling, M. (2012). Media governance and corporate social responsibility of media organizations: an international comparison. Business Ethics: A European Review, 21(2), 154167. https://doi.org/10.1111/j.1467-8608.2011.01646.x

Lee, S. Y. \& Riffe, D. (2017). Who sets the corporate social responsibility agenda in the news media? Unveiling the agenda-building process of corporations and a monitoring group. Public Relations Review, 2(43), 293-305. https://doi.org/10.1016/j.pubrev.2017.02.007

López-Cepeda, A. M. (2012). Modelos audiovisuales públicos en España. Perfil profesional, empresarial y político de sus principales órganos internos de gestión. Comunicación y Sociedad, 25(1), 399-427. https://core.ac.uk/download/pdf/83577869.pdf 
López-Cepeda, A. M. \& Manfredi, J. L. (2013). Análisis de la transparencia de las páginas web de los principales medios de comunicación audiovisuales en España. Trípodos, 1(32), 45-62. http:// www.tripodos.com/index.php/Facultat_Comunicacio_Blanquerna/article/view/78

Mediaset España (2016). Informe de responsabilidad corporativa 2015. http://servicios.telecinco. es/inversores/MEDIASET_informe_2015/descarga/Mediaset_descarga_2015_esp_03.html

Medina, M. \& Ojer, T. (2009). Valoración del servicio público de televisión. Comparación entre la BBC y TVE. Revista latina de comunicación social, 64, 275-294. https://doi.org/10.4185/RLCS64-2009-823-275-299

Moneva, J. M. (2005). Información sobre responsabilidad social corporativa: Situación y tendencias. RAE: Revista Asturiana de Economía, 34, 43-67. https://dialnet.unirioja.es/servlet/ articulo? codigo $=2232918$

Morales-Blanco-Steger, B., \& Fuente-Cobo, C. (2018). Confianza de las audiencias en las marcas televisivas: propuesta de indicadores de responsabilidad social y reputación corporativas. El profesional de la información, 27(3), 537-547, https://doi.org/10.3145/epi.2018.may.07

Moreno, Á. \& Capriotti, P. (2006). La comunicación de las empresas españolas en sus webs corporativas. Análisis de la información de responsabilidad social, ciudadanía corporativa y desarrollo sostenible. Zer-Revista de Estudios de Comunicación, 11(21), 47-62. http://www.ehu. eus/ojs/index.php/Zer/article/view/3718

Núñez-Ladeveze, L., Irisarri, J.A. \& Morales, B-S.B. (2015). La responsabilidad social propia de las empresas de televisión de amplia cobertura: contenidos de riesgo. Comunicación y Sociedad. 28(3), 65-82. https://dadun.unav.edu/handle/10171/39270

Orozco, J. \& Ferré Pavia, C. (2011). Los stakeholders de las empresas de comunicación en el ámbito de la responsabilidad social corporativa. Folios, 25, 107-125.

Orozco Toro, J. A. (2017). La incidencia de la RSC en la imagen corporativa. Revista Internacional de Cultura Visual, 4 (1). http://journals.epistemopolis.org/index.php/imagen/article/view/82.

Radio Televisión Española (2016). Memoria de servicio público y responsabilidad social corporativa 2015. http://www.rtve.es/contenidos/corporacion/MEMORIA_SP_Y\%20RSC_2015.pdf

Sánchez, A. (2001). El comportamiento ético en las empresas de comunicación. En M. Codina (ed.), De la ética desprotegida: ensayos sobre deontología de la comunicación. Navarra: Eunsa, 21-39.

Sandoval, M. (2013). Corporate social (ir)responsibility in media and communication industries. Javnost-ThePublic, 20(3), 39-57. https://doi.org/10.1080/13183222.2013.11009120

Sierra, V. D., \& Pascual, C. O. (2012). RSC en el sector de la televisión: un estudio longitudinal de las memorias de sostenibilidad. aDResearch: Revista Internacional de Investigación en Comunicación, (6), 112-129.

Sweeney, L. \& Coughland, J. (2008). Do different industries report Corporate Social Responsibility differently? An investigation through the lens of stakeholder theory. Journal of Marketing Communications, XIV (2), 113-124. https://doi.org/10.1080/13527260701856657

Tapia, A. \& Caerlos, R. (2014). La exposición de información corporativa en página web: el caso de las empresas del IBEX35. Sphera Publica, 2(14), 155-175. http://sphera.ucam.edu/index.php/ sphera-01/article/view/233 
Truñó Gual, J. I. \& Rialp Criado, J. (2008). La responsabilidad social corporativa: Gestión empresarial de un activo intangible. Revista de Contabilidad y Dirección, 7, 163-184. https:// dialnet.unirioja.es/servlet/articulo?codigo $=3127478$

Tuñez-Lopez, M., \& Costa-Sanchez, C. (2018). European Public Broadcaster Interaction and Social Media Policy to Public Value Management. Prisma Social, (22), 61-90.

Vila-López, N.; Küster-Boluda, I.; Bigné, E. (2013). Credibilidad corporativa en cadenas televisivas: antecedentes y efectos. Revista española de investigación en marketing ESIC, 17 (1), 61-82. https://doi.org/10.1016/S1138-1442(14)60019-X

Villafañe, J. (2004a). La buena reputación. Claves del valor intangible de las empresas. Madrid: Pirámide.

Villafañe, J. (2004b). El estado de la publicidad y el corporate en España y Latinoamérica. Madrid: Pirámide.

World Business Council for Sustainable Development (2002). Corporate Social Responsibility. Génova: The WBCSD's Journey. 